\title{
Study of Test Case Selection and Prioritization
}

\author{
Kevilienuo Kire \\ Lovely Professional University \\ Phagwara \\ Punjab
}

\author{
Neha Malhotra \\ Lovely Professional University \\ Phagwara \\ Punjab
}

\begin{abstract}
Software testing is very important for ensuring software quality in organizations. In fact, the quality of test suite plays an important role for the success of software testing .Ant colony optimization (ACO) which is a part of Swarm Intelligence is used to settle optimization problem. This paper attempts to provide ideas of Test Case Selection and Prioritization which Ant Colony Optimization which can be helpful for the researchers who are working on software testing. There is a great expectation in the field of Swarm Intelligence as it always comes with a challenging thought. $\mathrm{ACO}$ is one of such promising approaches for its application in Software Testing.
\end{abstract}

\section{General Terms}

Algorithms, Software Testing, Test Case, Prioritization

\section{Keywords}

Ant Colony Optimization, Coverage, Genetic algorithms, Simulated Annealing Algorithms, Pheromone Table, Regression Testing, Test Case Prioritization, Test Case Selection.

\section{INTRODUCTION}

In this world of technology, softwares have been used in every aspect like in business, organization, educational institution, games, home etc. We must focus on the quality of software. In order to achieve good quality software, software testing must be performed. For conducting the software testing, time and costs constraints must be considered.

To test the code there are different types of testing among which regression testing is one of it. After the coding is completed yet need arises to make some modifications, updating or deletion for adding new features and so on. The need to retest the test-suite arises for the purpose of maintaining the software after any changes is made.

For testing purpose a number of test cases are generated, it is not possible to test all the test cases. We must perform the testing in such a way that the test suite or the test case must be effective; it must yield to the detection of faults as soon as possible. Regression test selection is a process that helps in reducing the number of test cases. Once we are done with the selection of test cases, we must execute it to find the bugs or faults. To make the testing more effective we introduce Regression test prioritization for detecting the faults earlier.

Effective Algorithms or techniques must be used in testing such that high coverage and fault detection ratio is achieved. Swarm Intelligence is an artificial intelligence; it is so called because swarms have the capability to solve problems. Swarm Intelligence Algorithms include Particle Swarm Optimization and Ant Colony Optimization.

In order to assist the software testing we have algorithms such as Genetic Algorithm, Simulated Annealing Algorithms and Particle Swarm Optimization have been widely used but when it comes to Ant Colony Optimization it is not still deeply studied in generating test case and prioritization as stated by Chengying Mao, Xining Yu1 et al. in the paper "Generating Test Data for Structural Testing Based on Ant Colony Optimization".

\section{REVIEW OF LITERATURE}

Chengying Mao have reformed the basic Ant Colony Optimization to generate better quality test data for the purpose of earlier fault- revealing.Four rules have been redefined to achieve the objective namely: the local transfer rule, the global transfer rule and pheromone update rule. The criterion used is the branch coverage. The result of the reformed Ant Colony Optimization outperforms the algorithms namely: Simulated Annealing and genetic algorithm [1].

Rui Ding proposed an algorithm by applying particle swarm optimization into genetic algorithm, to overcome the limitation of genetic algorithm that is local convergence. They have used classical triangle problem and the result is compared with the original genetic algorithm and it resulted that the proposed algorithm yields better efficiency and better test cases [2].

Osman Gokalp reformed the original Ant Colony Algorithm by employing crossover mechanism in genetic algorithm. Four ant colonies have been employed rather than one colony and it also employs pheromone tables .Each colony will have their own pheromone tables and the best pheromone tables with best solution is chosen. The reformed algorithm is tested using Traveling Salesman Problem. The result of the reformed algorithm outperforms the original Ant Colony Algorithm [3].

Nirmal Kumar Gupta have proposed a strategy that uses genetic algorithm to reduce the number of unfeasible test cases and establishing genetic algorithm to generate suitable test cases and hence improving the genetic algorithm .The genetic algorithm is used to generate the test cases for Object Oriented Software that is for java programs. But practically to use genetic algorithm there is a problem as there is local convergence problem [4].

Wang Jun proposed test case prioritization by using genetic algorithm, which is regression-based testing, such that faults can be revealed more quickly, increase the software reliability and also save computational time and resources [7].

K. Karnavel used the Bee Colony Optimization (BCO) algorithm and performed regression testing and prioritization. The fault coverage is incorporated with the BCO algorithm for fault revealing within less execution time. Using this approach to test the software application, it is found that the testing cost and the execution time have been reduced [8].

Geniana Ioana Laiu used the evolutionary algorithms to generate path test data. The evolutionary algorithms are namely Genetic Algorithm (GA), Simulated Annealing (SA) and Particle Swarm Optimization (PSO).The test data generated from those algorithms are compared to find out 
which algorithms produces better quality of test data. The result shows that Simulated Annealing produces better quality of test data than Particle Swarm Optimization and Genetic Algorithm [11].

Daniel Di Nardo discussed an industrial study of the Coverage-Based Test Case Prioritization. Four different prioritization techniques are examined and evaluated .The coverage-based prioritization techniques are namely: Total Coverage, Additional Coverage, Total Coverage of Modified Code, and Additional Coverage of Modified Code. The result is that the additional coverage with finer grained coverage criteria such as block, basic block and decision are better than the other mentioned coverage-based prioritization techniques [12].

Luciano S. de Souza. Proposed a multi-objective Particle Swarm Optimization (PSO) for selecting the test cases based on functional requirements coverage and execution effort. Binary Multi-Objective PSO (BMOPSO) and Binary MultiObjective (PSO) algorithms are implemented. The result is found to be better than the random search approach for executing the test cases. They have also cited that future work can be performed based on the same experiment but with higher number of test suites [14]

Bharti Suri implemented Ant Colony Optimization Algorithm for Test Case Selection and Prioritization. In their study, have used a tool ACO_TCSP for implementation. The best solution was not found by them for all cases but the results closely related to the optimal solution. There is a reduction in the test suite size which is achieved to be $62.5 \%$ in all the 4 test runs. Future work emphasis to apply the tool on more examples to prove the effectiveness of the proposed technique [15].

Minjie Yi proposed an algorithm for path oriented testing by combining two algorithms namely: Ant Colony System Algorithm and Genetic Algorithm (ACSGA) and the proposed algorithm is used to generate path oriented test data. The problem used is Triangle discrimination problem. The result of the proposed algorithm is then compared with the Genetic Algorithm and it is found that the proposed algorithm is more efficient than genetic algorithm [17].

\section{PROBLEM FORMULATION}

\subsection{Existing system}

The Existing system ,test case prioritization using Genetic Algorithm, Simulated Annealing Algorithms and Particle Swarm Optimization have been widely used but when it comes to Ant Colony Optimization it is not still deeply studied in generating test case and prioritization. It is also stated by Changing Mao, Xinxiang Yu1 in the paper "Generating Test Data for Structural Testing Based on Ant Colony Optimization".

Moreover in regression testing when the code changes we have to retest even those successfully executed test suites to check whether the changes have introduced new bugs or faults .As a result the problem here is that there is time consuming and extra efforts and resources needed[6].

\section{COMPARISON OF ACO WITH GA,SA AND PSO.}

1. In real time the ACO can adapt to changes and it is better than Simulated Annealing and Genetic Algorithm for problems like Travelling Salesman Problem where the graph changes dynamically.
2. ACO outperforms simulated annealing and genetic algorithm for the metrics namely average coverage (AC), successful rate (SR) and average convergence generation (AG).

3. In genetic algorithm, every iteration produces a generation of individuals. Practically, the computation time must not be infinite, so there is a need to limit the generation of individuals. After limiting the generations, the solution that we get may locate in unwanted paths as a result failed to get the global optimum. It is not suitable for global search. [4]

4.The limitation of Bee Colony Optimization is that it is complicated to map the waggle dance to the solution, it requires deep knowledge of mathematics and one must have the knowledge of factors such as waggle dance, food source quality etc.

5. Premature convergence is the limitation of Particle Swarm Optimization (PSO) and GA.

Table 1 Experimental results of Algorithms for Test Data Generation [1].

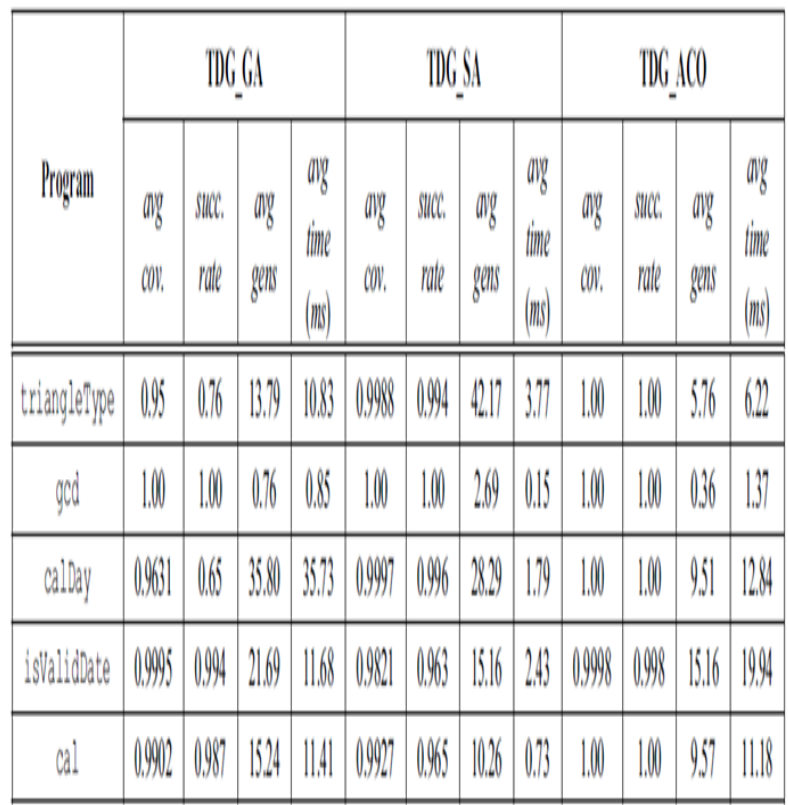

\section{CONCLUSIONS}

Present work surveyed on various techniques of Software Test Case Selection and Prioritization in Ant Colony Optimization as well as brief comparisons of ACO algorithm with GA,SA and PSO algorithims.ACO has been successfully applied to many NP-hard combinatorial optimization problems especially to traveling salesman problem (TSP).As the quality of test data set has a great impact on the software testing researchers can focused more in ACO for reducing the time of global/local search and pheromone update.

\section{ACKNOWLEDGMENTS}

From the enormous of my heart I thank my mentor Neha Malhotra for the continuous support and for her patience, motivation, enthusiasm, and for her immense knowledge. Without her valuable suggestions, it is not possible for me to carry out with the study. 


\section{REFERENCES}

[1] Chengying Mao, YuXinxin, Chen Jifu, Chen Jinfu (2012)"Generating Test Data for Structural Testing Based on Ant Colony Optimization "12th International Conference on Quality Software, Xi'an, Shaanxi, pp. 98 101

[2] Ding Rui, Feng Xianbin, Li Shuping, Dong Hongbin (2012) "Automatic Generation of Software Test Data Based on Hybrid Particle Swarm Genetic Algorithm", IEEE Symposium on Electrical \& Electronics Engineering (EEESYM), Mudanjiang, Kuala Lumpur, pp. $670-673$.

[3] Gokalp Osman and Ugur Aybars (2012) "Improving Performance of ACO Algorithms Using Crossover Mechanism Based on Mean of Pheromone Tables". International Symposium on Innovations in Intelligent Systems and Applications (INISTA), Trabzon, pp. $1-4$.

[4] Gupta Nirmal Kumar and Rohil Mukesh Kumar (2013) "Improving GA based Automated Test Data Generation Technique for Object Oriented Software", IEEE International Advance Computing Conference (IACC), Ghaziabad, pp.249 - 253.

[5] Jun Wang, Yan Zhuang, Chen Jianyun (2011) "Test Case Prioritization Technique based on Genetic Algorithm", International Conference on Internet Computing and Information Services, Hong Kong, pp. 173 - 175.

[6] Karnaveland K and Santhoshkumar J (2013) “Automated Software Testing for Application Maintenance by using Bee Colony Optimization algorithms (BCO)", International Conference on Information Communication and Embedded Systems (ICICES), Chennai, pp. 327 330.

[7] [9] Kan Stephen H (2002).Metrics and Models in Software Quality Engineering, Second Edition, Addison Wesley.
[8] Kothari, C.R (2004).Research Methodology, Methods and Techniques, 2nd Revised edition, New Age International Publisher, Jaipur, pp.348-350.

[9] Latiu Geniana Ioana, Cret Octavian Augustin, Vcariu Lucia (2012) "Automatic Test Data Generation for Software Path Testing using Evolutionary Algorithms", International Conference on Emerging Intelligent Data and Web Technologies, Bucharest, pp.1-8.

[10] Nardosdd Di Daniel, Alshahwan Nadia, Briand Lionel, Labiche Yvan (2013) "Coverage-Based Test Case Prioritization: An Industrial Case Study", IEEE Sixth International Conference on Software Testing, Verification and Validation", Luembourg, pp. $302-311$.

[11] Musa John D (2004).Software Reliability Engineering: More Reliable Software Faster and Cheaper,2nd. Edition, JWOMINGTON, IND1ANA47403.

[12] Souza Luciano S. de, Miranda Pericles B. C. de , Prudencio Ricardo B. C., Barros Flavia de A. (2011) "A Multi-Objective Particle Swarm Optimization for Test Case Selection Based on Functional Requirements Coverage and Execution Effort",23rd IEEE International Conference on Tools with Artificial Intelligence, Boca Raton, FL,pp. 245 - 252

[13] Suri Bharti, Singhal Shweta (2011)" Implementing Ant Colony Optimization for Test Case Selection and Prioritization" International Journal on Computer Science and Engineering (IJCSE), India, pp.1924-1932.

[14] Suri Bharti, Singhal Shweta (2012)" Literature Survey of Ant Colony Optimization in Software Testing" CSI Sixth International Conference on Software Engineering (CONSEG), Indore, pp.1-7.

[15] Yi Minjie (2012),"The Research of path-oriented test data generation based on a mixed ant colony system algorithm and genetic algorithm", International Conference on Wireless Communications, Networking and Mobile Computing (WiCOM), Shanghai , pp.1-4. 